\title{
Effect of stem reserve mobilization on grain filling under drought stress conditions in recombinant inbred population of wheat
}

\author{
Ashutosh srivastava ${ }^{*}$, Puja Srivastava, Achla Sharma, R. S. Sarlach and N. S. Bains \\ ${ }^{1}$ Department of Botany, Punjab Agricultural University, Ludhiana-141004 (Punjab), INDIA \\ Department of Plant Breeding \& Genetics,Punjab Agricultural University, Ludhiana-141004 (Punjab), INDIA \\ ${ }^{*}$ Corresponding author. E-mail: aksri_du@yahoo.com \\ Received: April 12, 2016; Revised received: September 7, 2016; Accepted: January 1, 2017
}

\begin{abstract}
Pre-anthesis carbon assimilation of stem reserves is considered as an important source for grain filling during post anthesis drought stresses that inhibit photosynthesis. 175 RILs from cross (C518/2*PBW343) along with check cultivars were evaluated for stem reserve mobilization under irrigated and rainfed conditions. These two cultivars belonging to distinct adaptation mechanism, offer several morpho-physiological and biochemical contrasts. C 518 is tall and adapted to low input rainfed conditions whereas PBW 343 is semi-dwarf and input responsive. Further C 518 is known for better stem reserves on account of larger peduncle and strong commitment to grain filling due to effective stem reserve mobilization. The parents and the RIL population was tested for stem reserve mobilization by defoliation of flag leaf and second leaf at anthesis under irrigated and rainfed environments. Evaluated entries differed significantly $(p<0.001)$ for reduction in 1000 grain weight under defoliation $(T G W L)$. Percent reduction in 1000 grain weight ranged from $4.4 \%$ to $39.6 \%$ under irrigated environment and $3.2 \%$ to 35.0 under rainfed condition. A significant positive correlation $(r=+0.357)$ between stem reserve mobilization and peduncle length was observed under rainfed condition. Tested RILs vary individually for stem reserve mobilization when subjected to removal of flag leaf and second leaf inhibiting the photosynthesis. The genotypes with better stem reserve mobilization based on 1000 grain weight in the absence of photosynthesis may also provide relative tolerance to drought.
\end{abstract}

Keywords: Carbohydrates, Defoliation, Sink-source modification, Stem reserve mobilization, Wheat

\section{INTRODUCTION}

Wheat responses to drought stress are particularly acute during the grain-filling period because the grain filling period is very sensitive to plant water status. Several studies have shown that that post-anthesis water deficits result in early senescence and more mobilization of pre-anthesis stored assimilates to grains in cereals (Yang et al., 2003). Remobilization of stored carbohydrates reserves in wheat is accelerated by water stress and that water deficits imposed during grain-filling enhanced plant senescence and affects grain-filling by shortens grain-filling period and reduction in grain weight (Yang et al., 2001). Current photosynthesis is mainly depends on the active green surfaces of the plant that intercepts light for carbon assimilation. Effect of drought stress and natural senescence reduce the rate of photosynthesis at the terminal stage, whereas at this stage the need of photosynthesis by the growing kernel increases. In this situation, stem reserves play a vital source for grain filling and wheat crops in dry land areas may depend more on stem reserves than on current photosynthesis for proper grain filling (Ehdaie et al., 2006). A better stem reserve and its remobilization have been considered as a drought adaptive trait in wheat for drought tolerance (Reynolds et al., 1999). Maydup et al. (2010) reported that the removal of flag leaf significantly reduced the total grain weight per spike about $25 \%$ in wheat cultivars. It has been also shown in another experiments when a photosynthesis organ (flag leaf) of plant is removed, the compensations in the remaining photosynthesis tissues or remobilization may occur through stem reserve and diminish the photo assimilate reduction (Chanishvili et al., 2005).

Several studies has been validate that when severe drought stress occurs at the time of grain filling, the desiccation of leaves greatly reduces photosynthesis and in this situation, grain filling mainly depends on mobilized stem reserves (Bidinger et al., 1977; Davidson and Birch, 1978; Hunt, 1979; Blum, 1988). Improving the capacity for supporting grain filling by stem reserves is an important breeding target in cereals subjected to environmental stresses during grain filling. Blum (1983a, b) proposed the use of chemical desiccation treatment of the canopy at anthesis as means for inhibiting plant photosynthesis and thus signify the capacity for grain filling by stem reserves. The treatment does not induce drought stress, but simulates the effect of stress by inhibiting current assimi- 


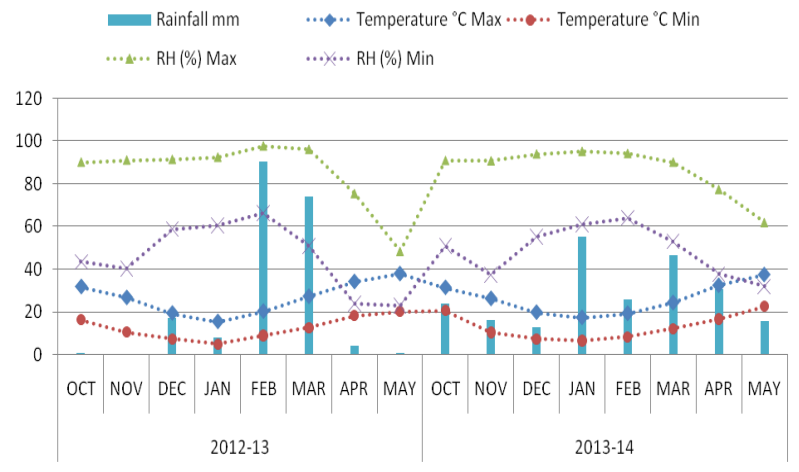

Fig. 1. Agrometerological data during crop seasons 2012-13 and 2013-14.

lation. Flag leaf is the most important photo-assimilate supplier for growing grains and about 30 to $50 \%$ of the assimilates for grain filling contributes by flag leaf photosynthesis in wheat and initiation of grain filling coincides with the onset of senescence, therefore, photosynthesis of the flag leaf is the most important basis of the formation of grain yield and it is interesting to evaluate changes in grain weight of different wheat cultivars when just the flag leaf is detached from the stem (Zhang et al., 2006).

Grain filling in wheat depends on two major sources of carbon, namely, current photosynthesis in leaves and mobilization of stored water-soluble carbohydrates (WSCs) from the stem internodes into the growing grains (Ehdaie et al., 2006). Stem WSCs in wheat consist mainly of fructans, followed by sucrose and usually lesser amounts of glucose and fructose. In contrast to other cereals, such as maize and rice, wheat stores very little starch in the stems. The contribution of remobilized stem WSC reserves to grain filling becomes more important when current photosynthesis is reduced by partial defoliation (Borras et al., 2004). It has been shown that the contribution of stem reserves in grain yield are different in different studies due to environmental conditions and the use of different wheat cultivars and it is varied from 6 to $100 \%$ (Blum, 1998). The tolerant cultivars have more ability to store assimilates and they have greater efficiency in transforming these reserves under stress condition (Ezzat-Ahmadi et al., 2011). So investigating the remobilization of stem reserves in wheat cultivars can be considered as a tool for selecting the drought tolerance.

These two cultivars PBW 343 and C 518 belongs to distinct adaptation groups, offer several morphophysiological and biochemical contrasts. C518 is tall and adapted to low input rainfed conditions whereas PBW 343 is semi-dwarf and input responsive. Further C518 is known for better stem reserves on account of larger peduncle and strong commitment to grain filling due to effective stem reserve mobilization (Bala et al., 2010). In the above view, present investigation was carried out to assess the genetic differences, among recombinant inbred population of wheat lines (C 518/2*PBW 343) for potential of stem reserve mobilization under drought conditions.

\section{MATERIALS AND METHODS}

Field experiments were carried out at experimental area of Department of Plant Breeding and Genetics, Punjab Agricultural University Ludhiana $\left(30^{0}-54^{\prime} \mathrm{N}\right.$ and $75^{\circ}-48^{\prime}$ E, $247 \mathrm{~m}$ altitude). The conducted experiment of specific RIL population was developed by cross of (C518/2*PBW 343). During first experiment (2012-13), plant material consisted of 700 RILs including parents (PBW 343 and C 518) and experimental design was randomized block design with two replications. Plots were $1 \mathrm{~m}$ per row spaced $0.2 \mathrm{~m}$ distanced. Initially, population was evaluated for morphophysiological traits of individual RIL. Data were recorded for days to anthesis, canopy temperature, chlorophyll content, plant height, peduncle length, 1000 grain weight and grain yield (data not shown).

During second experiment (2013-14), A small subset of 175 RILs reconstituted from first year experiment on the basis of yield percent reduction, along with parents (PBW 343 and C 518) and 19 check viz., commercial cultivars for timely sown irrigated (PBW 621 and

Table 1. Analysis of variance for peduncle length and 1000 grain weight under irrigated and rainfed conditions.

\begin{tabular}{|c|c|c|c|c|c|c|c|}
\hline \multirow{3}{*}{ Source of variation } & \multirow{3}{*}{ DF } & \multicolumn{6}{|c|}{ Mean square of characters } \\
\hline & & \multicolumn{3}{|c|}{ Irrigated } & \multicolumn{3}{|c|}{ Rainfed } \\
\hline & & PL & TGW & TGWL & PL & TGW & TGWL \\
\hline Replication & 2 & 11.66 & 73.287 & 2.158 & 0.288 & 24.302 & 4.275 \\
\hline Block within rep (adj) & 39 & 3.23 & 6.707 & 10.505 & 2.275 & 5.901 & $15.00 * *$ \\
\hline Component B & 39 & 3.23 & 6.707 & 10.505 & 2.275 & 5.901 & 15.00 \\
\hline Treatment (unadj) & 195 & 110.45 & 101.93 & 79.975 & 112.680 & 85.030 & 67.668 \\
\hline Treatment (adj) & 195 & $105.57 * *$ & $95.45 * *$ & $74.99 * *$ & $104.64 * *$ & $80.79 * *$ & $65.05^{* *}$ \\
\hline Intra Block Error & 351 & 2.698 & 8.305 & 10.432 & 2.921 & 6.612 & 5.347 \\
\hline Randomized complete Block Error & 390 & 2.752 & 8.145 & 10.439 & 2.857 & 6.541 & 6.313 \\
\hline Total & 587 & 38.56 & 39.523 & 33.511 & 39.330 & 32.676 & 26.688 \\
\hline Efficiency relative to RCBD & & 100.32 & 98.06 & 100 & 97.78 & 98.92 & 110.92 \\
\hline $\mathrm{R}^{2}$ & & 0.958 & 0.874 & 0.814 & 0.956 & 0.879 & 0.880 \\
\hline CV (\%) & & 4.44 & 8.04 & 9.84 & 4.65 & 7.26 & 7.09 \\
\hline
\end{tabular}

Abbreviations: PL -Peduncle length, TGW- 1000 grain weight, TGWL- 1000 grain weight under defoliation treatment 
Ashutosh srivastava et al. / J. Appl. \& Nat. Sci. 9 (1): 1-5 (2017)

Table 2. Performance of selected inbred lines along with check cultivars under irrigated and rainfed conditions.

\begin{tabular}{|c|c|c|c|c|c|c|c|c|c|c|}
\hline \multirow[b]{2}{*}{ RILs } & \multicolumn{5}{|c|}{ Irrigated } & \multicolumn{5}{|c|}{ Rainfed } \\
\hline & PL & TGW & TGWL & \% Red & $\%$ SR & $\mathbf{P L}$ & TGW & TGWL & \% Red & $\% \mathrm{SR}$ \\
\hline 30 & 29 & 34.34 & 30.35 & 11.6 & 88.3 & 32 & 33.64 & 31.25 & 7.1 & 92.8 \\
\hline 32 & 33.5 & 45.07 & 38.18 & 15.3 & 84.7 & 33 & 40.37 & 36.52 & 9.5 & 90.4 \\
\hline 41 & 39 & 42.36 & 36.85 & 13.0 & 86.9 & 35 & 40.27 & 37.53 & 6.8 & 93.2 \\
\hline 47 & 36.5 & 39.27 & 35.92 & 8.5 & 91.4 & 35 & 38.56 & 35.05 & 9.1 & 90.8 \\
\hline 80 & 31.5 & 41.75 & 38.04 & 8.9 & 91.1 & 28 & 35.45 & 33.73 & 4.9 & 95.1 \\
\hline 84 & 42.5 & 40.55 & 36.65 & 9.6 & 90.3 & 35 & 39.25 & 37.07 & 5.6 & 94.4 \\
\hline 108 & 37.5 & 39.89 & 36.99 & 7.3 & 92.7 & 39 & 40.71 & 38.32 & 5.9 & 94.1 \\
\hline Rils-Min & 24 & 21.44 & 20.47 & 4.4 & 53.9 & 23 & 21.03 & 19.41 & 3.2 & 50.9 \\
\hline Rils- Max & 54.5 & 45.38 & 42.83 & 39.6 & 96.7 & 50.5 & 42.89 & 41.11 & 35.0 & 95.4 \\
\hline Rils-Mean & 36.8 & 35.44 & 32.4 & 11.0 & 83.2 & 34.8 & 34.94 & 32.29 & 10.4 & 82.1 \\
\hline PBW 343 & 29.7 & 37.03 & 32.19 & 13.1 & 86.9 & 28 & 35.53 & 31.91 & 10.2 & 89.0 \\
\hline PBW 621 & 29.8 & 36.95 & 32.21 & 12.8 & 87.2 & 27 & 32.98 & 28.38 & 13.9 & 86.1 \\
\hline C 518 & 43 & 42.29 & 37.62 & 11.0 & 88.9 & 40.5 & 39.75 & 37.27 & 6.2 & 93.8 \\
\hline PBW 644 & 36 & 43.08 & 39.45 & 8.4 & 91.6 & 35.7 & 41.59 & 38.87 & 6.5 & 93.5 \\
\hline PBW 527 & 30.3 & 34.68 & 29.79 & 14.1 & 85.9 & 28.5 & 33.23 & 28.75 & 13.5 & 86.5 \\
\hline C 306 & 45.6 & 43.8 & 39.57 & 9.7 & 90.3 & 46 & 42.57 & 39.3 & 7.7 & 92.3 \\
\hline C 273 & 46.8 & 39.89 & 35.35 & 11.4 & 88.6 & 49 & 38.37 & 36.74 & 4.2 & 95.7 \\
\hline C 591 & 47.5 & 43.44 & 39.02 & 10.2 & 89.8 & 41.5 & 42.39 & 40.66 & 4.1 & 95.9 \\
\hline C 286 & 46.8 & 42.35 & 38.36 & 9.4 & 90.6 & 43.5 & 39.67 & 35.96 & 9.4 & 90.6 \\
\hline C 281 & 47.3 & 39.51 & 36.36 & 8.0 & 92 & 41 & 38 & 35.42 & 6.8 & 93.2 \\
\hline C 285 & 54.3 & 40.66 & 36.78 & 9.5 & 90.4 & 43 & 38.06 & 35.97 & 5.5 & 94.5 \\
\hline PBW 706 & 25.3 & 42.68 & 38.09 & 10.8 & 89.3 & 37.5 & 39.72 & 37.57 & 5.4 & 94.6 \\
\hline PBW 175 & 41.3 & 44.46 & 37.42 & 15.8 & 84.2 & 38.5 & 40.8 & 34.92 & 14.4 & 85.6 \\
\hline PBW 691 & 36.5 & 42.26 & 36.58 & 13.4 & 86.5 & 32.3 & 37.78 & 35.04 & 7.3 & 92.7 \\
\hline BWL 1856 & 36.3 & 44 & 37.18 & 15.5 & 84.5 & 34.5 & 42.8 & 39.39 & 8.0 & 92 \\
\hline HD 2967 & 36.7 & 42.88 & 37.61 & 12.3 & 87.7 & 32 & 34.22 & 27.35 & 20.1 & 79.9 \\
\hline Kirchauff & 31.3 & 37.34 & 31.83 & 14.8 & 85.2 & 36.5 & 35.23 & 30.98 & 12.1 & 87.9 \\
\hline Babax & 34.8 & 33.45 & 30.4 & 9.1 & 90.9 & 33.5 & 30.98 & 25.22 & 18.6 & 81.4 \\
\hline Excalibur & 28.7 & 34.18 & 31.97 & 6.5 & 93.5 & 28.5 & 29.41 & 24.85 & 15.5 & 84.5 \\
\hline Gladius & 37.2 & 38.07 & 33.6 & 11.7 & 88.3 & 37.8 & 37.81 & 34.81 & 7.9 & 92.1 \\
\hline Drysdale & 36.8 & 32.84 & 29.33 & 10.7 & 89.3 & 35 & 25.76 & 23.13 & 10.2 & 89.8 \\
\hline
\end{tabular}

Abbreviations: PL -Peduncle length, TGW- 1000 grain weight, TGWL- 1000 grain weight under defoliation treatment, \% RedPercent of reduction, \% SR- percent of stem reserve mobilization.

HD 2967), rainfed cultivars (PBW 644, PBW 527, PBW 175), traditional tall cultivars (C 306, C 273, C 591, C 286, C 281, C 285), advance breeding lines (PBW 706, PBW 691, BWL 1856) and Australian drought cultivars (Kirchauff, Babax, Excalibur, Gladius, Drysdale). The experimental design was square lattice with three replications. Plots were $1 \mathrm{~m}$ long with 4 rows spaced $0.2 \mathrm{~m}$ apart. The drought environment was created by withholding irrigation and created temporary rain shelter over trails whenever required. Agrometerological data recorded during crop period from Agrometerological station, PAU, Ludhiana, Punjab (Fig. 1). Normal recommended agronomic practices for growing timely sown wheat crop were followed.

Defoliation treatments: Defoliation treatments (removal of flag leaf and penultimate leaf from stem) were applied to five tagged tillers from different plants with uniform height and spike size before onset of anthesis. Another five tillers were tagged and not defoliated, which were used as control for the experiment. At maturity, all the tillers were separately harvested, the spikes were threshed individually and the grains weight was recorded. Stem reserves were estimated based on percent reduction of thousand kernel weight for each line. "Percent reduction in kernel weight" by defoliation treatment was obtained by comparing mean kernel weight under defoliation with mean kernel weight in the controls, for each tested genotype (Blum, 1998). At appropriate stage of growth three plants were randomly selected from each row of each plot and data were collected for days to anthesis, days to maturity, peduncle length and 1000-grain weight. The analysis of variance was computed by SAS (version 9.2) and correlation analysis among different traits was analyzed.

\section{RESULTS AND DISCUSSION}

The analysis of variance (Table 1) for peduncle length and 1000 grain weight was highly significant $(p<0.001)$ due to defoliation (removal of flag leaf and penultimate leaf) under both irrigated and rainfed environments, indicating the existence of sufficient genetic variability among the population. Previously, Regan et al. (1993) have significant differences in the response of genotypes to defoliation treatment. Genetic variability among genotypes for tested traits may be helpful for yield improvement. The RIL population showed a 
Table 3. Correlation analysis among various traits under irrigated and rainfed conditions.

\begin{tabular}{lccccc}
\hline & PL & GFP & TGW & TGWL & $\%$ SRM \\
\hline PL & 1 & $0.281^{* *}$ & $0.404^{* *}$ & $0.496^{* *}$ & $0.307^{* *}$ \\
GFP & $0.279^{* *}$ & 1 & $0.245^{* *}$ & $0.289^{* *}$ & $0.101^{*}$ \\
TGW & $0.402^{* *}$ & $0.302^{* *}$ & 1 & $0.911^{* *}$ & -0.002 \\
TGWL & $0.478^{* *}$ & $0.304^{* *}$ & $0.928^{* *}$ & 1 & $0.407^{* *}$ \\
\%SRM & $0.357^{* *}$ & $0.127^{* *}$ & $0.213^{* *}$ & $0.558^{* *}$ & 1
\end{tabular}

- -Rainfed, - -Irrigated, PL-Peduncle length, GFPGrain filling period, TGW- Thousand grain weight, TGWLThousand grain weight under defoliation, SRM- \% Stem reserve mobilization.

range of variants for both peduncle length and 1000 grain weight. Under irrigated conditions, PBW 343 and $C 518$ had peduncle length of $29.7 \mathrm{~cm}$ and $43.0 \mathrm{~cm}$ respectively (Table 2). Under rainfed conditions, peduncle length was recorded for PBW $343(28.0 \mathrm{~cm})$ and C $518(40.5 \mathrm{~cm})$. It has been indicated that more the peduncle length, more stem reserve mobilization resulting in effective movement of carbon assimilates from source (stem) to sink (grain) in absence of leaves. Stem reserve mobilization based on percent reduction in 1000 grain weight showed differential response under both environments. Percent of stem reserve mobilization under irrigated condition ranged between $53.9 \%$ to $96.7 \%$ with an average of $83.2 \%$ among the RILs, whereas parents showed PBW 343 (86.9\%) and C 518 (88.9\%). Maximum percent SRM was found in RIL 108 (92.7\%), followed by RIL 47 (91.4\%), RIL 80 (91.1\%) and RIL 84 (90.3\%). However, among check cultivars, maximum \% SRM was found in Excalibur (93.5\%), PBW 644 (91.6 \%), Babax (90.9), C 286 (90.6\%), C 285 (90.4\%) and C 306 (90.3\%). In rainfed conditions, percent SRM ranged between 50.95 to $95.4 \%$ with an average of $82.1 \%$ among the RILs, while parents showed PBW $343(89.0 \%)$ and C 518 (93.8\%). Maximum \% SRM was recorded for RIL 80 (95.1\%) followed by RIL 84 (94.4\%), RIL 108 (94.1 \%), RIL 41 (93.2) and RIL 30 (92.8\%). Among check cultivars, maximum percent SRM was recorded for C 591 (95.9\%) followed by C 273 (95.7 \%), PBW 706 (94.6\%), C 285 (94.5\%), C 518 (93.8 $\%$ ) and PBW 644 (93.5\%). It showed that percent of SRM higher under rainfed condition than irrigated condition. The genotype having minimum percent reduction exhibits high utilization of stem reserve under drought stress. The genotypes having high utilization of stem reserves were considered relatively drought tolerant as compared to those with low utilization (drought susceptible). Theoretically, genotypes which accumulate a large amount of water soluble carbohydrates (WSC) in the stem may be able to relocate more carbohydrates to the grain than genotypes with less stem carbohydrate concentrations when the supply by photosynthesis is limited.

Correlation analysis among various traits were analyzed and is presented in Table 3. Grain filling period was found positively correlated with TGW $\left(\mathrm{r}_{\mathrm{i}}=0.245\right)$, TGWL $\left(r_{i}=0.289\right)$ and SRM $\left(r_{i}=0.107\right)$ under irrigated condition. However, under rainfed conditions, it was found positively correlated with TGW $\left(r_{r}=0.302\right)$, TGWL $\left(r_{r}=0.304\right)$ and SRM $\left(r_{r}=0.127\right)$. Peduncle length showed positively correlated with GFP $\left(\left(\mathrm{r}_{\mathrm{i}}=\right.\right.$ $0.281)$, TGW ( $\left.r_{i}=0.404\right)$, TGWL $\left(r_{i}=0.496\right)$ and SRM $\left(r_{i}=0.307\right)$ under irrigated condition. However, under rainfed conditions, peduncle length was found positively correlated with GFP $\left(r_{r}=0.279\right)$, TGW $\left(r_{r}=\right.$ 0.402), TGWL $\left(r_{r}=0.478\right)$ and SRM $\left(r_{r}=0.357\right)$. Under irrigated environment, TGWL showed positive significant correlation with SRM $\left(r_{i}=0.407\right)$ while under rainfed condition, TGWL showed strong positive correlation with SRM $\left(r_{r}=0.558\right)$. Blum (1998) found a high correlation across five wheat cultivars between the reduction in kernel weight by chemical desiccation and the reduction in kernel weight by heat stress during grain filling. Stem reserve mobilization showed strong positive correlation among the all traits under rainfed condition than relatively irrigated condition. It showed that mobilization of pre anthesis stored carbohydrates higher under rainfed environment. Hunt (1979) and Borrell (1993) also reported that there is positive correlation between peduncle length and remobilization of stem reserves in wheat. The impact of stem reserves should be evaluated only under stress conditions, which equally inhibit crop assimilation during grain filling in all materials tested. In this study, on the basis of stem reserve under drought condition, the highest stem reserve mobilization was recorded in RIL 80 (95.1\%) followed by 84 (94.4\%), 108 (94.1\%) and $41(93.2 \%)$. Similar finding was also reported by Yang and Zhang (2006) who suggested that cultivars with high potential for remobilization in dry environments is of best practices to sustain high performance. Maydup et al. (2010) have reported that blockage of current photosynthesis with defoliation near anthesis stage makes a significant contribution to 1000 grain weight of wheat from $13 \%$ to $33 \%$ in control and $22 \%$ to $45 \%$ under water stress conditions. This indicates the role of stem reserve mobilization to grain filling under drought stress condition than under irrigated condition. Stem reserves from pre-anthesis plant assimilation are being increasingly recognized as an important source of carbon for grain filling, when current photosynthesis is inhibited by drought, heat or disease stress during this stage. Defoliation treatments aimed at decreasing photosynthesis in the whole plant may cause an increase in the photosynthetic rate of the remaining organs (Eyles et al., 2013). In fact, it has been reported that such defoliation treatments may trigger unwanted compensatory mechanisms in the remaining organs like peduncle of the plant to increase the contribution of assimilates reserves to grain filling (Schnyder, 1993; Plaut et al., 2004). 


\section{Conclusion}

Grain weight of some RILs decreased about to $36 \%$ in response to defoliation treatment at anthesis showing that wheat grain filling of these RILs are probably source limited. Hence it unable to contribute much assimilates through peduncle to grain filling. In nondefoliated plants, almost all of the sucrose had disappeared from the stems at maturity, but in the partially defoliated plants a substantial amount of sucrose remained in the lower stem internodes and in the peduncle. So, that the defoliation treatment in some way impaired the capacity of the plants to remobilize all of their stem reserves to developing grains and thus their ability to import sucrose. This might suggest that RILs 80, 84, 108 and 41 have higher strength in stem reserve mobilization under drought stress conditions and would be useful in further breeding programme.

\section{REFERENCES}

Bala, S., Asthir, B. and Bains, N.S. (2010). High temperature response leads to altered membrane permeability in conjunction with carbon utilization in wheat. Seed Sci Biotechnol., 4: 10-14

Bidinger, F., Musgrave, R.B. and Fischer, R.A. (1977). Contribution of stored preanthesis assimilates to grain yield in wheat and barley. Nature, 270: 431-433

Blum, A. (1988). Plant breeding for stress environments. CRC Press: Boca Raton, FL, pp. 232

Blum, A. (1998). Improving wheat grain filling under stress by stem reserve mobilization. Euphytica., 100: 77-83

Blum, A., Maye, J. and Golan, G. (1983b). Chemical desiccation of wheat plants as a simulator of post-anthesis stress. II. Relations to drought stress. Field Crops Res., 6: $149-155$

Blum, A., Poyarkova, H., Golan, G. and Mayer, J. (1983a). Chemical desiccation of wheat plants as a simulator of post-anthesis stress. I. Effects on translocation and kernel growth. Field Crops Res., 6: 51-58

Borras, L., Slafer, G.A. and Otegui, M.E. (2004). Seed dry weight response to source-sink manipulation in wheat, maize and soybean: a quantitative reappraisal. Field Crops Res., 86:131-146

Borrell, A., Incoll, L. and Dalling, M. (1993). The influence of the Rht1 and Rht 2 alleles on the deposition and use of stem reserve in wheat. Annals of Bot .,71: 317-326

Chanishvili, G., Badridze, S., Barblishivili, T.F. and Dolidze, M.D. (2005). Defoliation, photosynthetic rates, and assimilates transport in grapevine plants. Russian $J \mathrm{Pl}$ Physiol., 52:448-453

Davidson, J.L. and Birch, J.W. (1978). Responses of a stand- ard Australian and Mexican wheat to temperature and water stress. Aust J Agric Res., 29: 1091-1106

Ehdaie, B., Alloush, G.A., Madore, M.A. and Waines. J.G. (2006). Genotypic variation for stem reserves and mobilization in wheat: I. Post anthesis changes in inter node dry matter. Crop Sci ., 46: 735-746

Eyles, A., Pinkard, E.A., Davies, N.W., Corkrey, R., Churchill, K., O'Grady, A.P., Sands, P. and Mohammed, C. (2013). Whole-plant versus leaf-level regulation of photosynthetic responses after partial defoliation in Eucalyptus globulus saplings. J Exp Bot., 64:1625-1636

Ezzat-Ahmadi, M., Noormohammadi, G.H., Ghodsi, M. and Kafi, M. (2011). Effect on the accumulation and re-material sources of stress and limitation of photosynthesis in wheat genotypes. Iranian J Field Crops Res., 9: $241-229$

Hunt, L. (1979). Stem weight changes during grain filling in wheat from diverse sources. In S. Ramanujam (ed.) Proceed. 5th Int. Wheat Genetics Symposium, New Delhi, India. pp. 923-927

Maydup, M.L., Antonietta, M., Guiamet, J.J., Graciano, C., Lopez, J.R. and Tambussi, E.A. (2010). The contribution of ear photosynthesis to grain filling in bread wheat (Triticum aestivum L.). Field Crops Res., 119: 48-58

Plaut, Z., Butow, B.J, Blumenthal, C.S. and Wrigley, C.W. (2004). Transport of dry matter into developing wheat kernels and its contribution to grain yield under postanthesis water deficit and elevated temperature. Field Crops Res., 86: 185-198

Regan, K.L, Whan, B.R. and Turner, N.C. (1993). Evaluation of chemical desiccation as a selection technique for drought resistance in dry land wheat breeding program. Aust J Agric Res., 44: 1683-1691.

Reynolds, M.P., Skovamand, B., Trethowan, R. and Pfeiffer, W. (1999). Evaluating a conceptual model for drought tolerance. In: Using molecular markers to improve drought tolerance. (Ed.): J.M. Ribaut ,CIMMYT, Mexico.

Schnyder, H. (1993). The role of carbohydrate storage and redistribution in the source-sink relations of wheat and barley during grain filling. New phytologist, 123:233-245

Yang, J. and Zhang, J. (2006). Grain filling of cereals under soil drying. New Phytologist, 169:223-236

Yang, J., Zhang, J., Wang, Z., Zhu, Q. and Liu, L. (2001). Water deficit-induced senescence and its relationship to the remobilization of pre-stored carbon in wheat during grain filling. Agron J., 93: 196-206

Yang, J., Zhang, J., Wang, Z., Zhu, Q. and Liu, L. (2003). Involvement of abscisic acid and cytokinins in the senescence and remobilization of carbon reserves in wheat subjected to water stress during grain filling. Plant Cell Environ., 26:1621-1631 